\title{
Flux of meteoroid impacts on Mercury
}

\author{
S. Marchi ${ }^{1}$, A. Morbidelli ${ }^{2}$, and G. Cremonese ${ }^{3}$ \\ 1 Dipartimento di Astronomia, Vicolo dell'Osservatorio 2, 35122 Padova, Italy \\ e-mail: marchi@pd.astro.it \\ 2 Observatoire de la Côte d'Azur, Nice, France \\ e-mail: alessandro.morbidelli@obs-nice.fr \\ 3 INAF - Osservatorio Astronomico di Padova, 35122 Padova, Italy \\ e-mail: cremonese@pd.astro.it \\ Received 5 August 2004 / Accepted 18 October 2004
}

\begin{abstract}
In this work we present a new estimate of the meteoroid flux on Mercury. The flux has been obtained as a result of material delivery from both the 3:1 and $v_{6}$ resonances, and calibrated on the basis of the Earth meteoroid flux. Our model does not take into account non-gravitational forces, like the Poynting-Robertson effect, and for this reason it is suitable for bodies having dimension greater than about $1 \mathrm{~cm}$. We also analyse the dependence of the predicted impactor flux on Mercury on meteoroid size. Possible asymmetries of the meteoroid flux over the surface (morning/evening hemispheres) and along the orbit (perihelion/aphelion) are also discussed.
\end{abstract}

Key words. planets and satellites: individual: Earth - planets and satellites: individual: Mercury - meteors, meteoroids

\section{Introduction}

The impact flux on Mercury (as well as on other planets) is the consequence of several different physical processes providing bodies on planetary crossing orbits. Detailed studies for the Earth have shown that the range of sizes impacting our planet span more than eight order of magnitude: from $\mu \mathrm{m}$ up to hundreds of meters. There is no reason to doubt that the same is true also for the other terrestrial planets. Such a flux of material onto Mercury has several effects, such as the formation of craters and the well-known "maturation" of the soils. Moreover, and most importantly for this study, the effect of the meteoroid flux on Mercury's exosphere is not yet clear.

The exosphere refers to the tenous part of an atmosphere where collisions among volatile species become negligible. In the case of Mercury, the exobase, namely the limit under which the collisions start to be important, is actually represented by its surface. This means that the sources and sinks of the exosphere are strongly linked to the composition and structure of the surface. A fraction of volatiles released into the exosphere is thought to be produced by impact vaporization of meteoritic material. On the basis of existing models, this fraction is estimated to be in the range of 10-20\% (Killen et al. 2001) to $100 \%$ (Morgan et al. 1988). In the second hypothesis, all volatiles come from meteoritic material. Unfortunately, the meteoritic gardening and the impacts rate on Mercury's surface are very difficult to determine because of several unknowns and variables related to the composition of the surface and the flux of meteoroids. The meteoroid flux used in the literature for Mercury studies are roughly derived from estimates at the Earth heliocentric distance. This means we may not have a good estimate of the statistics on the number of impacts and the velocity distribution of the meteoroids. Cintala (1992) dealt with micrometeoroid impacts, but his work was restricted to sizes less that $1 \mathrm{~mm}$ and it cannot be extrapolated to larger bodies. Indeed, meteoritic flux on Mercury depends on the particle size, because meteoroids of different sizes follow different dynamical evolution. Meteoritic sizes smaller than about $1 \mathrm{~cm}$ are particles with a dynamical evolution dominated by the PoyntingRobertson effect. On the other hand, particles having a larger size follow a completely different dynamical evolution. Most of the large meteoroids arriving on the terrestrial planets come from two important resonances located in the asteroid main belt (the 3:1 and the $v_{6}$ ).

The study of the exosphere is one of the reasons why we started studying meteoroid flux on Mercury. In this paper we shall describe our results, which, considering that Mercury is the target of two important space missions to be launched in 2004 (Messenger) and 2012 (BepiColombo), will be useful for the mission studies and the observation strategies. Another interesting issue we shall address concerns the asymmetry of the number of impacts on the surface of Mercury, which could be related to short and long term variations of the exosphere's observed intensity.

\section{The model}

Generally speaking, the meteoroid flux on Mercury represents the number of bodies impacting on the planet per unit time. Since the effects of impacts are mainly dependent on the energy 
involved (and hence on the radius $r$ and impact velocity $v$ of meteoroids), it is convenient to describe the flux in terms of these variables by using the differential flux $\phi(v, r)$. Thus, the meteoroid flux $\Phi$ can be written as follows:

$\Phi=\iint \phi(v, r) \mathrm{d} v \mathrm{~d} r=\iint f(v, r) h(r) \mathrm{d} v \mathrm{~d} r$

where we have explicitly indicated the dependence of the differential flux $\phi$ on the differential normalized impact velocity distribution $f(v, r)$, and the number of impacts, $h(r)$, over the whole surface of Mercury, per unit of time and unit radius. The delivery routes from the main belt are the well known resonances. Among them, the most efficient in ejecting material toward the inner solar system are the 3:1 and $v_{6}$ (see Morbidelli \& Gladman 1998; Bottke et al. 2002). For this reason we limit our analysis to the delivery from these resonances. Our approach to the problem is very similar to that of Morbidelli \& Gladman (1998), for the Earth's meteoroids. We have used, as a basis, the numerical simulations of test particles initially placed in the $v_{6}$ secular resonance or in the 3:1 mean motion resonance with Jupiter, described in Bottke et al. (2002). These integrations account for the perturbations of the planets, from Venus to Neptune, and followed the dynamical evolution of thousands of particles from their initial location in either of these resonances until their ultimate fate. The typical dynamical endstates for the particles were (in order of decreasing likelihood) collision with the Sun, ejection from the solar system and collision with a planet. Because the Poynting-Robertson drag (see for instance Burns et al. 1974) was not included in these simulations, the dynamical results should be considered valid for particles larger than approximately $1 \mathrm{~cm}$ in radius. The simulations did not account for the direct perturbations exerted by Mercury. This might seem surprising for a study on the bombardment of Mercury, but it is in fact a reasonable approximation, for two reasons. First, the perturbations exerted by Mercury do not change significantly the dynamics of the particles, at least in a statistical sense (Ito \& Malhotra 2004). Second, in order to compute the collision rate of the particles with Mercury, we do not monitor the number of impacts in the simulation (this procedure would suffer from small number statistics even if Mercury were present in the simulations), but instead we use, as in Morbidelli \& Gladman (1998), an Öpik-Wetherill semianalytic calculation (Öpik 1976; Wetherill 1967). The latter is done as follows. The numerical simulations give a time history of the orbital elements $a, e$ and $i$ for each particle. For each set $(a, e, i)$, the collision probability with Mercury and the average impact velocity are computed averaging over all possible orbital configurations occurring during a precessional cycle of the orbits and taking into account Mercury's gravitational focusing. For Mercury we have assumed an orbit with fixed values $a=0.387 \mathrm{AU}, e=0.2$ and $i=7^{\circ}$, uniformly precessing in time. In practice, the calculations has been done using a code developed by Farinella \& Davis (1992) and kindly provided to us. In order to calibrate the meteoroid flux on Mercury relative to that (measured) on the Earth, we have applied the same procedure to compute also the collision probability with our planet (assuming Earth's orbital elements $a=1 \mathrm{AU}$, $e=0.0167, i=0^{\circ}$ ), and the corresponding impact velocity.
So far, the results obtained do not depend on the size of the particles (apart from the requirement that the radii should be larger than $\sim 1 \mathrm{~cm}$ so that the dynamics are not significantly affected by the Poynting-Robertson drag). However, in order to obtain a correct evaluation of the flux of meteoroids to a planet, we must consider an important, size-dependent effect: meteoroids in space have a final physical lifetime due to collisions that break them into smaller pieces. The catastrophic collisional half-life of meteoroids that are crossing the main asteroid belt (i.e. bodies having perihelion distances, $Q$, greater than $1.8 \mathrm{AU}$ ) is estimated to be

$\tau_{\mathrm{c}}(r)=1.4 \times \sqrt{r} \mathrm{My}$

where $r$ is the particle radius in $\mathrm{cm}$ (Wetherill 1985; Farinella et al. 1998). This estimate for the collisional lifetime holds also in more refined collisional models, which account for a size-dependent specific energy of desruption, as derived in hydrocode experiments (Bottke et al. 2004). However, Bottke et al. (1994) showed that for meteoroids decoupled from the asteroid belt ( $Q<1.8 \mathrm{AU}$ ) the collisional half-life is increased by orders of magnitude, so that in practice it can be ignored. In principle collisions can both destroy and generate particles of any given size, the generation being due to the disruption of precursor particles of larger radius. By fitting the observed semi-major axis distribution of the observed fireballs, Morbidelli \& Gladman (1998) showed that disruption dominates over production, so that the net effect of collisions is to decimate over time a population of meteoroids of any given size. To take into account this size-dependent decimation effect, for each particle in our numerical simulations we keep track of the total time spent on orbits with $Q>1.8 \mathrm{AU}$. We call this time the "collisional age" $T$ of the particle. The collisional age increases with the integration time as long as $Q>1.8 \mathrm{AU}$, and it is frozen otherwise. In summary, from the numerical simulations and using the procedures described above, we obtain a file separately for the $v_{6}$ and 3:1 resonances. Each line of the file corresponds to one particle at a specific output timestep, and reports the current orbital elements of the particle, the corresponding collision probabilities and impact velocities with Mercury/Earth, and the particle's collisional age. The total number of lines in the files for the $v_{6}$ and $3: 1$ resonances were about $2 \times 10^{6}$ and $7 \times 10^{5}$, respectively. These data have been obtained from the numerical integration of the dynamical evolution of 3600 and 2136 particles, respectively. Then, when we want to compute, say, the average collision probability with a planet for particles of radius $r$ coming out of a resonance, we simply read the file corresponding to that resonance; we multiply the collision probability that we read in each line of the file by the probability $P_{\text {live }}=1 / 2^{T / \tau_{\mathrm{c}}(r)}$ that the particle with the collisional age $T$ survives intact; finally we sum up all the obtained numbers and we divide the result by the total number of particles used in the simulation from which the file has been built.

\section{Impact flux on Mercury}

As shown in the previous section (see Eq. (1)), the meteoroid flux can be obtained by knowing two differential distributions. 

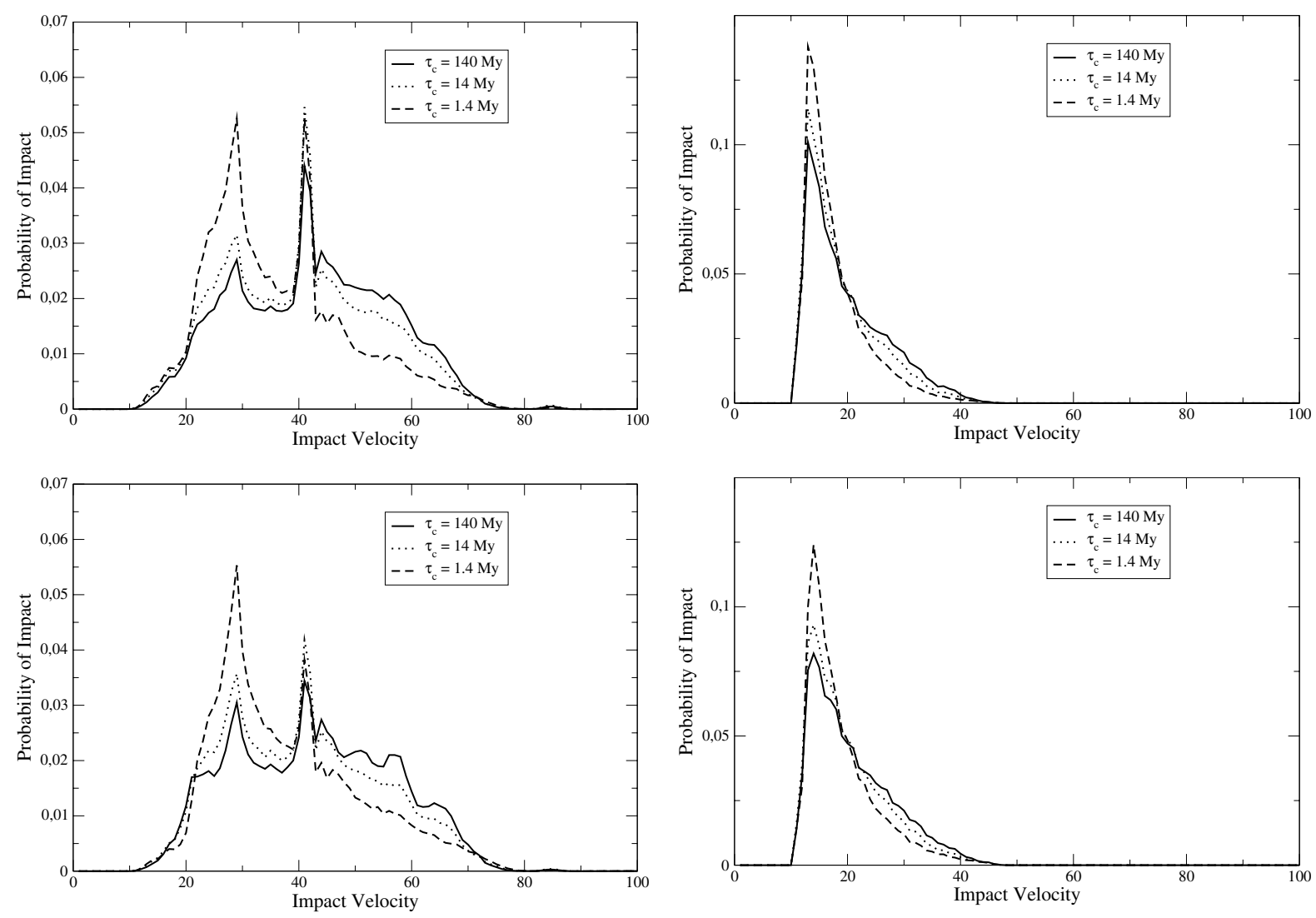

Fig. 1. Impact velocity distribution for Mercury (left panels) and Earth (right panels) for $\Gamma=1$ (upper panels), and $\Gamma=5$ (lower panels). Higher values of $\Gamma$ produce the same results as $\Gamma=5$, while $\Gamma<1$ is unlikely and hence not considered. Each panel shows distributions obtained for three values of $\tau_{\mathrm{c}}$ (see text for further details). The dependence of the distributions on $\tau_{\mathrm{c}}$ is not very strong. Thus, a modification in the collisional lifetimes reported in Eq. (2) would not significantly modify our results.

We deal first with the impact velocity distribution $f(v, r)$. In order to obtain the actual impact distributions on Mercury, it is necessary to "tune" the contribution of the two resonances to the total flux. In other words, we have to know the ratio between the rates at which objects are transported to the near-Earth space by the 3:1 and the $v_{6}$ resonance, respectively. Let $\Gamma$ be this ratio. The knowledge of $\Gamma$ is a necessary step because the impact's distribution from the two resonances could be much different from each other. $\Gamma$ is determined by all the processes which affect the delivery mechanism from the resonances (efficiency of dynamical perturbations, supply of bodies into the resonances, etc.), and hence cannot be evaluated a priori and with confidence. Morbidelli \& Gladman (1998) have estimated that $\Gamma \sim 5$, by fitting the semimajor axis distribution of the fireballs of chondritic origin, determined from the images of automatic camera networks (Wetherill \& ReVelle 1981; Halliday et al. 1996). However, given that this procedure also does not allow a precise determination of $\Gamma$, in the following we will also investigate how the results depend on $\Gamma$. In Fig. 1 (lower-left panel) the total fluxes on Mercury for $\Gamma=5$ are shown. The distributions reported are for different collisional life-times: 140, 14 and 1.4 My (namely for $r=10000$, 100 and $1 \mathrm{~cm}$, according to Eq. (2)) and they have been normalized to have area equal to 1 . With this choice the value $\int_{v_{\mathrm{i}}}^{v_{\mathrm{f}}} f(v, r) \mathrm{d} v$ represent the fraction of impacts having radius $r$ between $v_{\mathrm{i}}$ and $v_{\mathrm{f}}$. For comparison, Fig. 1 (lower-right panel) shows the Earth's impact distributions obtained with the same collisional life-times. The most important result is the wide range of impact velocities on Mercury: the mean impact velocity for all the distributions is about $30 \mathrm{~km} \mathrm{~s}^{-1}$, but their tails span from about 15 to $80 \mathrm{~km} \mathrm{~s}^{-1}$. For comparison, the Earth's impact distributions are much narrower (with a mean of about $20 \mathrm{~km} \mathrm{~s}^{-1}$ ) and with a maximum impact velocity of about $50 \mathrm{~km} \mathrm{~s}^{-1}$. Moreover, Mercury's impact distributions depend on the impactor sizes, i.e. the simplification $f(v, r)=f(v)$, used in some works (e.g. Cintala 1992), does not hold in our case. To quantify the effects of the impactor sizes, we note that the percentage of high velocity impactors (defined as those having $v>50 \mathrm{~km} \mathrm{~s}^{-1}$, namely the maximum impact velocity on Earth) are $31 \%, 25 \%$ and $19 \%$, respectively for $\tau_{\mathrm{c}}=140,14$, $1.4 \mathrm{My}$ (or $r=10000,100,1 \mathrm{~cm}$ ). It is worth to remind that indeed on Earth it is possible to have impacts with velocities up to $80 \mathrm{~km} \mathrm{~s}^{-1}$, but they are sporadic events related to retrograde swarm of fragments, presumably of cometary origin, which have not been considered in the present work. Moreover, we restrict our work to bodies having dimensions between 1 and $10000 \mathrm{~cm}$. Figure 1 also reports the distributions for $\Gamma=1$. This case implies that the 3:1 and the $v_{6}$ have the same "weight" in the production of planet-crossing bodies, while for $\Gamma=5$ the 3:1 resonance is 5 times more efficient. In both cases the impact distributions are almost the same, i.e. $\Gamma$ has only a slight influence on the impact distributions. Concerning the size 


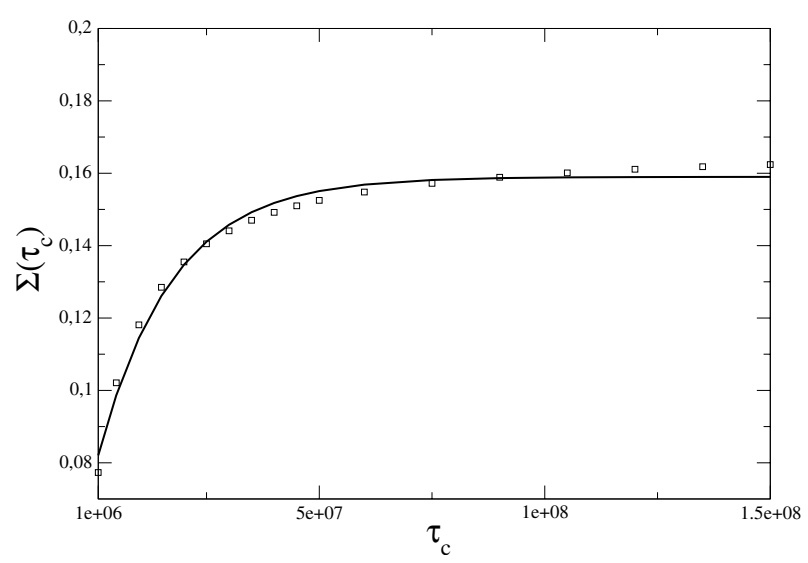

Fig. 2. Computed $\Sigma\left(\tau_{c}\right)$ (dots) and fitted distribution (solid line).

distribution of impactors on Mercury $h(r)$, as for $\Gamma$, it has to be calibrated with the flux observed on the Earth, for which we have reliable data (see Brown et al. 2002). Indeed, from our numerical simulations, we estimate the ratio $\Sigma$ of impacts on Mercury vs. the Earth for each projectile size, and we use this ratio to scale the impact rate with Mercury relative to that observed for the Earth.

We found that $\Sigma$ varies only by a few percent with $\Gamma$, while it depends on collisional lifetime (see Fig. 2). By a best fit we obtained the following expression for $\Sigma\left(\tau_{\mathrm{c}}\right)$ :

$\Sigma\left(\tau_{c}\right)=a\left(1-b \mathrm{e}^{-\tau_{c} / c}\right)$

where $a=0.159, b=0.511$ and $c=16.5 \mathrm{My} . \Sigma\left(\tau_{\mathrm{c}}\right)$ can be converted in $\Sigma(r)$ with the help of Eq. (2). In the light of this result, and using the expression derived from Brown et al. (2002), the differential size distribution of the bodies impacting the whole surface of Mercury can finally be written:

$h(r)=\frac{d}{r^{e}} \cdot \Sigma(r)$

where $r$ is the radius (in meters), $d=7.68$, and $e=3.7$. In Fig. 3 the differential distribution $h(r)$ for the Earth and Mercury are shown. Notice that, as expected, $\Sigma(r)$ has only a slight influence on the shape of $h(r)$, it reduces by about 1/10th the values for the Earth.

\section{Orbital and diurnal asymmetries}

The distributions reported in the previous section represent an average impact distribution on Mercury's orbit. However, since the orbit of Mercury is quite eccentric, we could expect some variation from the mean impact rate along its orbit. To test this possibility, we computed the distributions for two limiting cases. We considered two fictitious Mercuries, each having circular orbits with semimajor axis equal to Mercury's perihelion (resp. aphelion) and orbital velocity equal to that of the real Mercury at perihelion (resp. aphelion). The distributions obtained for the latter are almost the same as in the previous section and we do not show them. On the contrary, for the perihelion case, the impact distributions are quite different (see Fig. 4). In particular, we point out that for the perihelion case, the number of high velocity impacts is about $47 \%$,

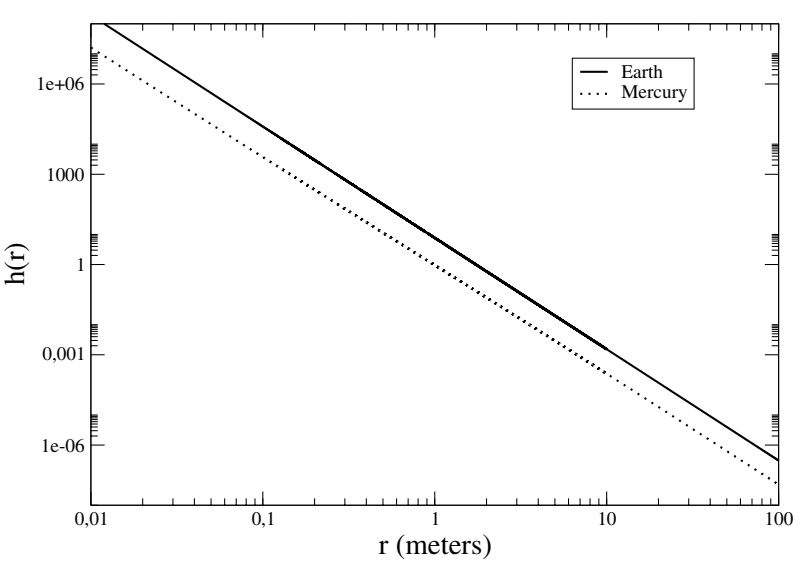

Fig. 3. Differential radius distribution, $h(r)$, for Mercury and the Earth, expressed in number of impacts over the whole surface per year per unit of impactor radius.
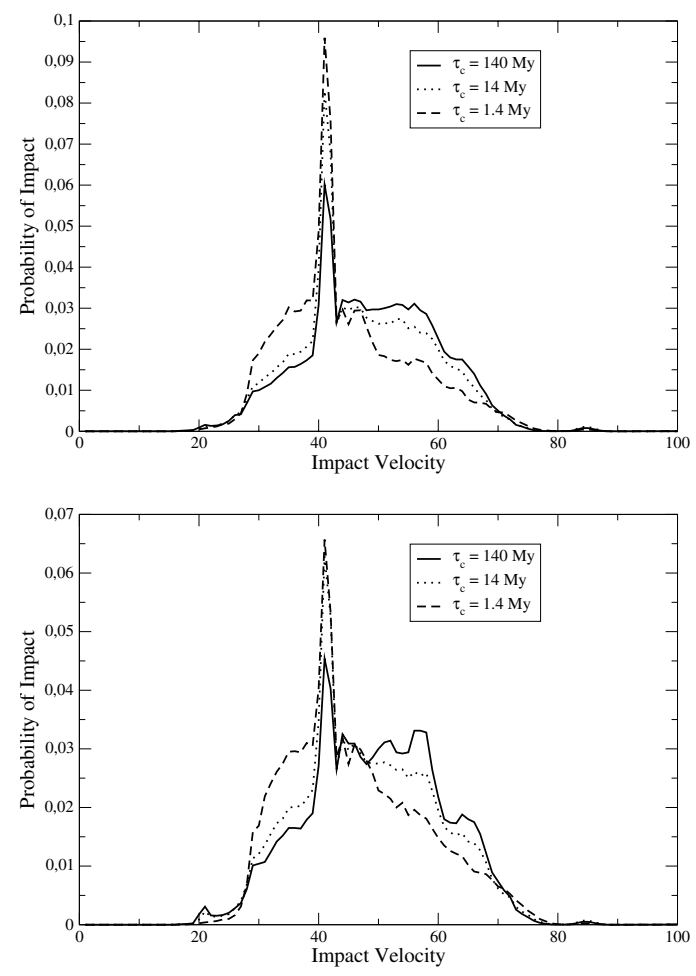

Fig. 4. Impact distribution on Mercury at perihelion for $\Gamma=1$ (upper panel) and $\Gamma=5$ (lower panel).

$43 \%$ and $33 \%$, respectively for $\tau_{c}=140,14$ and 1.4 My. Thus, impacts at perihelion happen at considerably greater velocity than the average case. Moreover, we also investigated possible asymmetries in the rate of impacts on the surface of Mercury. Asymmetries of impacts onto planets or satellites have been widely studied for synchronous rotating bodies (e.g. see Horedt $\&$ Neukum 1984; Marchi et al. 2004). For non-synchronous rotating bodies, like Mercury, the same considerations hold, but now the asymmetry is related to the morning-evening ( $\mathrm{am} / \mathrm{pm}$ ) hemispheres instead of to leading-trailing ones. Figure 5 shows this dependence for the three cases considered. As for the impact distributions, the ratio $\mathrm{am} / \mathrm{pm}$ depends on the collisional lifetime, but not much on $\Gamma$. In the average case the 


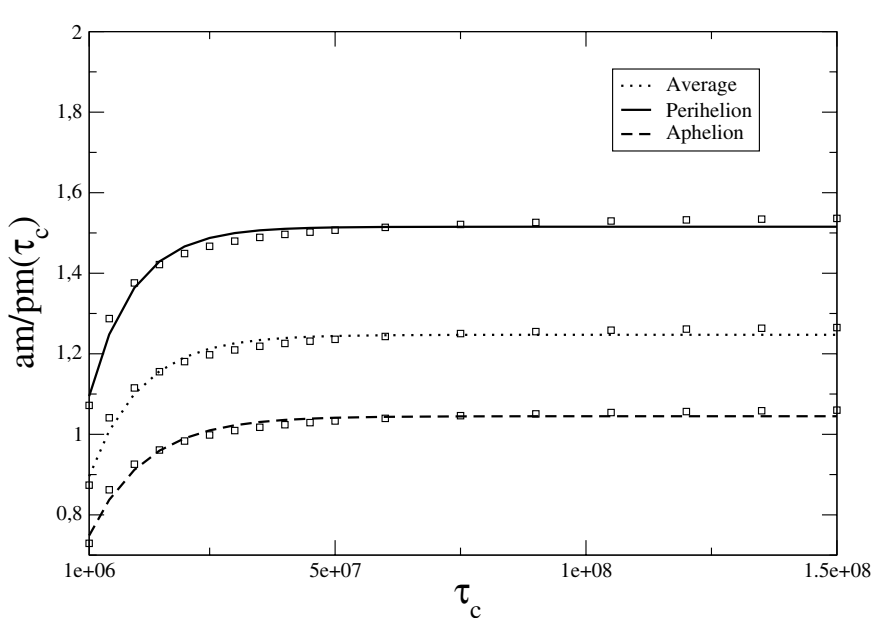

Fig. 5. Morning-evening impact asymmetry on Mercury's surface vs. collisional lifetime.

ratio $\mathrm{am} / \mathrm{pm}$ is greater than 1 except for very short collisional lifetimes (less then $5 \mathrm{My}$ ) which correspond to $r<13 \mathrm{~cm}$. Impacts at aphelion have a symmetric distribution over the surface $(a m / p m=1)$ at $\tau_{\mathrm{c}}=23$ My (i.e. $\left.r=270 \mathrm{~cm}\right)$ while at perhelion $a m / p m>1$ always. By best fitting, we obtained the folliwing expression for $\mathrm{am} / \mathrm{pm}\left(\tau_{\mathrm{c}}\right)$ :

$\operatorname{am} / p m\left(\tau_{c}\right)=f\left(1-g \mathrm{e}^{-\tau_{\mathrm{c}} / h}\right)$

where $f=1.25,1.51,1.04$ and $h=10.3,8.83,11.2 \mathrm{My}$, respectively for the average, perihelion and aphelion case; while $g=0.31$ for all cases. The increase of the $\mathrm{am} / \mathrm{pm}$ ratio with collisional lifetime is normal, as already pointed out by Morbidelli \& Gladman (1998). It is due to the fact that the longer the collisional lifetime, the more numerous are the meteoroids with small semi-major axis, which typically tend to fall on the morning hemisphere. Also, it is normal that the $\mathrm{am} / \mathrm{pm}$ ratio is larger for Mercury at perihelion, because the orbital velocity of the planet is higher, and the planet tends to catch up the meteoroids, rather than being caught up by them.

\section{Conclusions}

In this paper we dealt with the flux of meteoroids on Mercury in the range $1-10^{4} \mathrm{~cm}$. The range of dimensions considered here extends that previously investigated by Cintala (1992) from micro-meteoroids up to about $1 \mathrm{~cm}$. We limited our analyses to the contribution of asteroids to the flux, which, according to previous work concerning impacts on Earth (e.g. see Ceplecha 1992), should be dominant with respect to other components, like the cometary flux. However, this point deserves further study in light of the unexpectedly high number of comets seen by SOHO (see http://sohowww. nascom.nasa.gov/) near the Sun.

The findings of our work can be summarized as follows:

- Impacts on Mercury occur from 15 to $80 \mathrm{~km} \mathrm{~s}^{-1}$. For comparison, the maximum impact velocity on Earth is $50 \mathrm{~km} \mathrm{~s}^{-1}$. The impact velocity on Mercury in our model differs considerably from that of Cintala (1992): our distributions extend toward higher velocities, while that of
Cintala is much narrow and with a lower mean impact velocity of about $20 \mathrm{~km} \mathrm{~s}^{-1}$.

- $\Gamma$ has only a little influence on impact distributions. This result means that bodies delivered from the 3:1 and $v_{6}$ have about the same distribution of impact velocity.

- Impact distributions depend on the impactor sizes: the percentages of high velocity impactors (i.e. $v>50 \mathrm{~km} \mathrm{~s}^{-1}$ ) are $\sim 31 \%, \sim 25 \%$ and $\sim 19 \%$, respectively for $r=10000$, $100,1 \mathrm{~cm}$. The exact numbers depend on the esitmate of the collisional lifetime (see Eq. (2)).

- Impacts at perihelion happen at considerably greater velocity than impacts averaged over Mercury's entire orbit: the percentages of high velocity impacts are about $47 \%, 43 \%$ and $33 \%$, respectively for $r=10000,100,1 \mathrm{~cm}$.

- Impacts at aphelion have a symmetric distribution $(\mathrm{am} / \mathrm{pm}=1)$ for $r=270 \mathrm{~cm}$, while at perihelion is always $\mathrm{am} / \mathrm{pm}>1$. For Mercury's real orbit the ratio $\mathrm{am} / \mathrm{pm}$ is greater than 1 except for small impactors (i.e. $r<13 \mathrm{~cm}$ ). The maximum asymmetry is for large impacts at perihelion, for which we have $\mathrm{am} / \mathrm{pm}=1.5$, i.e. impacts on the morning hemisphere are $50 \%$ more numerous that those on the evening one.

The problem of volatile release from impacts and their contribution to the maintainance of Mercury's exosphere is rather complex and we shall deal with it in a subsequent paper. Here we stress that, even if most of the mass impacting the Earth daily (and presumably Mercury) is delivered by impactors in the size interval $10-200 \mu \mathrm{m}$, the range of projectile sizes that we have investigated in this paper could still be relevant for the exosphere problem because of their larger impact velocities relative to dust particles.

Acknowledgements. We thank the anonymous referee for comments that helped to improve the paper.

\section{References}

Bottke, W. F., Nolan, M. C., Greenberg, R., \& Kolvoord, R. A. 1994, in Hazards due to Comets and Asteroids, ed. T. Gehrels (Tucson, Arizona, USA: Univ. Arizona Press), 337

Bottke, W. F., Morbidelli, A., Jedicke, R., et al. 2002, Icarus, 156, 399

Bottke, W. F., Durda, D. D., Nesvorny, D., et al. 2004, Icarus, in press Brown, P., Spalding, R. E., ReVelle, D. O., Tagliaferri, E., \& Worden, S. P. 2002, Nature, 420, 294

Burns, J. A., Lamy, P. L., \& Soter, S. 1979, Icarus, 40, 1

Ceplecha, Z. 1992, A\&A, 263, 361

Cintala, M. J. 1992, JGR, 97, 947

Farinella, P., \& Davis, D. R. 1992, Icarus, 97, 111

Farinella, P., Vokrouhlicky, D., \& Hartmann, W. K. 1998, Icarus, 132, 378

Halliday, I., Griffin, A. A., \& Blackwell, A. T. 1996, M\&PS, 31, 185

Horedt, G. P., \& Neukum, G. 1984, Icarus, 60, 710

Ito, T., \& Malhotra, R. 2004, Icarus, submitted

Killen, R. M., Potter, A. E., Reiff, P., et al. 2001, JGR Planets, 106, 20509

Marchi, S., Barbieri, C., \& Lazzarin, M. 2004, P\&SS, 52, 671

Morbidelli, A., \& Gladman, B. 1998, M\&PS, 33, 999

Morgan, T. H., Zook, H. A., \& Potter, A. E. 1988, Icarus, 75, 156

Öpik, E. J. 1976, Interplanetary Encounters (New York: Elsevier)

Wetherill, G. W. 1967, JGR, 72, 2429

Wetherill, G. W., \& Revelle, D. O. 1981, Icarus, 48, 308 